\title{
FUENTES BIBLIOGRÁFICAS PARA LA HISTORIA EN INTERNET. ESTADO DE LA CUESTIÓN
}

\author{
MARÍA CRUZ RUBIO LINIERS \\ CINDOC-CSIC
}

RESUMEN: Se presenta un estado de la cuestión sobre las fuentes bibliográficas para la Historia existentes en Internet y su tipología: principales catálogos vituales, ediciones electrónicas de libros y repositorios de documentos, directorios de revistas impresas y eléctrónicas, así como las principales bases de datos historiográficas tanto generales como especializadas por épocas y temas. Se analiza y valora dicha oferta en razón a su accesibilidad y gratuidad. El movimiento internacional Open Access representa un avance muy positivo para el acceso a la información histórica. Las nuevas tecnologías están permitiendo a través de bibliotecas virtuales y bases de datos de contenido realizar metábusquedas de gran operatividad y buenos resultados. Se incluye una relación selectiva de dichos recursos con las direcciones eléctronicas y datos informativos.

PAlabras Clave: Fuentes de información histórica. Bibliografías. Catálogos. Bases de datos. Internet.

ABSTRACT: We offer an overview of the current state of web-based bibliographic resources for bistory along with a typology: the main virtual catalogues, e-books and collections of documents, guides to printed journals and e-journals, as well as the main historiographical databases, both general and specialised according to periods and themes.Each site is assessed according to accessability and user-friendliness. The international movement, Open Access, represents a major advance in access to bistorical information. Through access to virtual libraries and databases, the new technologies allow for manageable and highly useful megasearches. Included is a selective guide to these resources with full information and e-addresses.

KEY WORDS: Sources of historical information. history resources. bibliographies. Catalogues. Databases. Internet. 


\section{CARACTERÍSTICAS DE LA BIBLIOGRAFÍA DE HiSTORIA}

Para que exista una verdadera eficacia en la transmisión de la información es necesario conocer las características de la disciplina en la que se trabaja, su crecimiento, evolución, grado de interdisciplinariedad, el lenguaje que le es propio, así como el tipo de documentos que utiliza y publica el investigador. La Historiografía tiene ciertas peculiaridades documentales con entidad y carácter propio, y otras comunes con la mayoría de las Ciencias Sociales.

Los historiadores utilizan el documento de archivo como fuente primaria de su investigación, y por tanto es lógico que valoren sobre todo dichos fondos. Consideramos en historiografía fuentes primarias aquellas que se crearon en la época que se investiga y que representan el soporte documental de un estudio y el concepto de documento y documentación ha ido unido para el historiador a la documentación de archivo.

Hoy los archivos están empezando a despegar en la utilización de las nuevas tecnologías de la información. Internet permite no solo consultar los catálogos sino incluso llegar al documento. Pero no debemos olvidar que las fuentes primarias van mucho más allá de la documentación archivística sobre todo en Historia Contemporánea. Los libros, las revistas, los periódicos, las cartas, los programas de TV, la información que navega por Internet, serán un día fuentes primarias para la investigación histórica.

En un futuro no muy lejano las NTI no serán solo simples herramientas para la investigación histórica, sino que llegarán a convertirse en objeto de investigación. Ya ahora Internet no solo es un medio de comunicación e información sino también un modo de movilización política y social y por ello una fuente para el estudio de la historia del tiempo presente. Porque Internet también es ya Historia.

Varios son los problemas que tradicionalmente han hecho difícil el acceso y la recuperación de la producción bibliográfica. En primer lugar, y siendo la Historia una ciencia acumulativa, su obsolescencia es muy inferior a otras disciplinas y en muchos casos publicaciones «clásicas» continúan utilizándose y citándose. Además, la información de interés para los historiadores se encuentra tradicionalmente dispersa en repertorios bibliográficos y bases de datos de disciplinas sociales cercanas.

El peculiar ámbito de estudio de los historiadores el ser social en el tiempo, vuelve impreciso e indefinido su límite disciplinar y encuadra mal las publicaciones en los tradicionales sistemas de clasificación científica. Este carácter se acentúa en Historia Contemporánea, donde hasta un 20 o 30\% de la documentación válida en la búsqueda bibliográfica aparece en Bases de datos y catálogos de otras disciplinas cercanas como la Sociología o las Ciencias Políticas. Esta interdisciplinariedad afecta sobre todo a la recuperación en catálogos de bibliotecas por el «encasillamiento» de las materias y la difícil delimitación de la producción historiográfica, debido a los lenguajes clasificatorios y precoordinados de las bibliotecas. 
La mayor facilidad de consulta a los archivos nacionales y el indudable mayor interés del historiador por el pasado de su lugar, le lleva a investigar sobre un ámbito social, político y geográfico concreto, a utilizar una terminología y una periodización específica y propia. Por eso las historiografías nacionales y locales, tienen difícil proyección en ámbitos internacionales. Esto provoca una «endogamia» editorial con muy bajo porcentaje de historiadores que publican fuera de nuestro país. Con Internet sucede todavía lo mismo. Los grandes sistemas de información bibliográfica son de ámbito anglosajón, con escasa presencia de publicaciones españolas.

La tipología de los soportes en que publica el historiador es muy variada. Al año aparecen en nuestro país, más de 200 tesis doctorales, 2.000 monografías y 3.000 artículos de revistas de tema histórico, Sólo la base de Datos ISOCHISTORIA, contiene unas 70.000 referencias de artículos de revistas nacionales, desde 1975 a la actualidad.

Conviene recordar que hasta ahora el historiador ha utilizado preferentemente y en muchos casos únicamente como obra de consulta y publicación el libro, mientras que en otras Ciencias Sociales se da mayor importancia a las revistas. La razón fundamental ha podido ser hasta ahora la baja calidad de las publicaciones periódicas y el utilizarse este medio entre los historiadores para presentar sólo avances de trabajos que culminan en monografías. Las dificultades de publicar en forma de libro y la indudable mejora de la calidad de las revistas de Historia deben, como ya ha sucedido fuera de nuestras fronteras, cambiar métodos y usos, a favor de la agilidad y rapidez de difusión de las investigaciones a través de las revistas ${ }^{1}$.

¿Cual es la situación actual sobre la presencia de las fuentes bibliográficas de Historia en Internet?. La evolución de uso, cantidad y calidad de recursos ha tenido en los últimos años un crecimiento extraordinario. Esto es detectable a partir de nuestra primera aproximación al tema ${ }^{2} \mathrm{y}$ otros trabajos pioneros ${ }^{3}$.

En la actualidad dar una relación exhaustiva de dichos recursos resulta imposible, no solo por la enorme proliferación de catálogos y bibliografías existentes en la red, sino sobre todo por la «inestabilidad» de la información. Lo que

1 Esta tendencia ha sido impulsada a partir de las políticas nacionales de normalización y evaluación de revistas, que en nuestro pais tienen su máximo exponente en las investigaciones realizadas en el CINDOC (CSIC)

2 Rubio Liniers, María Cruz; Ruiz Franco, Rosario; Andres Verdú, Rosario de; Bustelo TORTELLÁ, Jaime: «Internet y la Historia de España». Revista General de Información y Documentación, 1998, 8 (2):149-163

3 Martínez de Velasco Farinós, Ángel: «Internet e Historia Contemporánea de España», Espacio, Tiempo y Forma. Historia Contemporánea, 1995, vol. 8, pp. 331-388. CANALES, Esteban: «Internet para historiadores», Revista Biblioteca Informacions, noviembre, 1996, pp. 9-11. LOPEZ YEPES, José. «Las Bases de datos históricas». Anales de Documentación, 1998, 1: 99-124. Gines HuerTas, Francisca: «Información, Historia e Internet». Revista d'Historia Medieval. 1998, (9): 297-312. PORRAS ARbOledAS, Pedro Andrés: «El Medievalismo en Internet». Medievalismo. Boletín de la Sociedad Española de Estudios Medievales, 1997, 7, (7):343-361. 
hoy existe, mañana puede haber desaparecido. Por ello, debemos centrarnos en sistemas ya consolidados y con ciertas garantías institucionales, y siempre con un carácter selectivo. Además, ya hoy estamos en condiciones de poder analizar en gran medida los principales recursos y presentar más que una simple guía o directorio un estado de la cuestión valorativo sobre el tema.

Las direcciones que aquí presentamos ${ }^{4}$ se han seleccionado teniendo en cuenta parámetros de calidad de presentación, gratuidad en el acceso, volumen de información disponible y garantías de la institución responsable del web.

\section{El ACCeso A Los Libros}

\subsection{Catálogos de bibliotecas}

Es en el ámbito de las bibliotecas donde mejores recursos podemos encontrar para la localización de libros. La casi totalidad de ellas tienen sus O'Pacs en Internet de forma gratuita y han creado además redes de catálogos colectivos. Pero por otra parte, al no haber sido concebidos como redes de información sino más bien de conservación, sus instrumentos de análisis —clasificaciones y encabezamientos de materia-, no resultan, a la hora de la recuperación temática, todo lo efectivos que desearían los especialistas. En España, las bibliotecas universitarias y sobre todo las científicas (Catálogo Colectivo del CSIC) tienen mejores posibilidades de búsqueda y recuperación que la Biblioteca Nacional., que sin embargo sigue siendo punto imprescindible de consulta para cualquier historiador, por el volumen de sus fondos. En cuanto a las bibliotecas extranjeras, su importancia reside obviamente en el ámbito geográfico de nuestra investigación.

A partir de los buscadores (Google, Altavista, etc..) y de los principales directorios que existen en Internet podemos acceder a todas las bibliotecas del mundo. La presencia cada vez mayor de catálogos colectivos de ámbito internacional en Internet hace más sencilla la búsqueda gracias a protocolos y metabuscadores que permiten la recuperación simultánea en un gran número de bases de datos.

Son las bibliotecas nacionales, garantes del almacenamiento de los fondos de cada país, las que mayor volumen de información ofrecen, aunque las posibilidades de consulta varían en función de los softwares de recuperación, campos de búsqueda y profundidad de análisis. Especialmente amigables son el catálogo de la Library of Congress o de la British Library.

Especial interés tienen los catálogos colectivos de bibliotecas, tanto nacionales como universitarios y científicos. En este ámbito podemos encontrar ver-

4 Al final de este artículo incluimos una lista comentada y ordenada por epígrafes según la tipología documental aquí desarrollada, de las direcciones que consideramos más interesantes. 
daderos macrocatálogos como el caso del de la Universidad de Karlruhes. La utilización del protocolo Z 39-40 está permitiendo el intercambio de registros entre bibliotecas, a través de un interfaz común que permita acceder a un gran número de catálogos del mundo simultáneamente.

Existen catálogos colectivos de ámbito autonómico de gran interés para la investigación histórica regional y local, como puede ser el caso del catálogo colectivo "Fonts d'Historia Local»" ${ }^{6}$, imprescindible para la Historia de Cataluña, y otros catálogos regionales como el de la Fundación Sancho el Sabio ${ }^{7}$, el catálogo colectivo de Euskadi para la Historia del País vasco ${ }^{8}$, o el catálogo del Centro de Estudios de Castilla La Mancha9

En los últimos años observamos una auténtica transformación de dichos catálogos en fuentes de información especializada, al «vaciar» artículos de revistas y actas de Congresos. Un buen ejemplo es la participación de un buen número de catálogos de bibliotecas universitarias en la base de datos Dialnet ${ }^{10}$ de la Universidad de la Rioja.

\subsection{Catálogos comerciales}

Los catálogos de editoriales y libreros, tanto de obras antiguas, como de libreros especializados o editoriales públicas y universitarias, son una de las formas más utilizadas por los historiadores para estar al día de las últimas novedades en su tema de investigación.

No olvidemos que un catálogo de librero ${ }^{11}$, fue un auténtico catálogo nacional, y aún hoy es una de las fuentes bibliográficas retrospectivas más importantes para localizar la literatura publicada en el siglo XIX y principios del XX.

Internet presenta un gran número de enlaces a librerías especializadas en Historia, tanto en fondo actual en venta como libros desclasificados. Especial interés son aquellas librerías virtuales como Amazon que permiten además «hojear» las obras, ya que nos presentan índices y capítulos e incluso referencias cruzadas a otros libros sobre el tema.

Algunos editores, como Marcial Pons ${ }^{12}$ en España, presentan además de sus catálogos, bibliografías seleccionadas por temas: «La Historia en femenino», «Trafalgar»o «Las Cruzadas».

http://www.ubka.uni-karlsruhe.de/kvk_span.html

$6 \mathrm{http}: / /$ www.diba.es/fonshl/fonshl.asp? cont $=157$

7 http://www4.gipuzkoa.net/corporac/cul/kulturaldi/index.asp

8 http://www.euskadi.net/r33-2522/es/

9 http://www.uclm.es/ceclm/bases.htm

10 http://dialnet.unirioja.es/

11 Palau y Dulcet, Antonio. Manual del librero hispanamericano. Bibliografía general española e hispano-americana desde la invención de la imprenta hasta nuestros tiempos. Barcelona:Librería Anticuaria de A.Dulcet, 1948-, 28 vols. +7 vols. de índices.

12 http://www.marcialpons.es 


\section{LA EDICIÓN ELECTRÓNICA. E-BOOKS Y REPOSITORIOS DE DOCUMENTOS}

En los últimos años están proliferando en Internet, depósitos de documentos y proyectos de digitalización de libros. Aunque para Historia la mayoría de los textos son documentos fuente, y los proyectos más importantes de libros electrónicos se refieren a obras literarias, también podemos encontrar un gran número de artículos de investigación que los propios autores han enviado a estos repositorios, bien como pre-prints, bien ya publicados en soporte papel. Importantes instituciones, universidades o empresas, están potenciando este recurso como un medio enormemente efectivo de difundir internacionalmente la producción historiográfica. Un ejemplo puede ser el repositorio de historiografía medieval Reti Medievali13, creado por varias universidades italianas que publican textos y materiales previamente evaluados (peer-reviewed) por su redacción.

El movimiento Open Access ${ }^{14}$, que defiende el acceso abierto y sin restricciones a la información a través de la publicación electrónica, es favorable a eliminar la obligatoriedad de cesión del copyright de los artículos publicados. Además de que la edición digital supone un $80 \%$ de ahorro respecto a la versión impresa permite además:

1. El incremento considerable de la difusión de los trabajos y por tanto hace llegar el conocimiento científico a todos los lugares del mundo donde tengan una conexión a Internet.

2. La máxima visibilidad para los autores de los artículos y una mayor internacionalización y difusión de sus trabajos

3. La máxima rapidez en la edición de los mismos.

4. ermite a los usuarios leer, imprimir, buscar y enlazar los textos completos de los artículos sin coste alguno, manteniéndose una garantía del control de la propiedad intelectual.

Hay disciplinas e instituciones científicas, sobre todo en el ámbito de las Ciencias Puras y Sociales, que han apostado por el Open Access. Por ahora la dispersión de los textos electrónicos es muy grande, ya que podemos encontrar trabajos en páginas personales o de instituciones, en programas de cursos, en webs de departamentos, faltando sobre todo en nuestro país grandes «archivos» que garanticen el mantenimiento y la actualización de estos fondos.

\footnotetext{
13 http://www.retimedievali.it/

14 http://www.geotropico.org/Berlin-I-2.pdf (Leída el 10-6-2005)
} 


\section{LAS PUBLICACIONES PERIÓDICAS EN INTERNET. EL ACCESO A REVISTAS.}

Series, revistas y periódicos, son cada vez más un importante vehículo de difusión de la investigación histórica. Los títulos de series y revistas son localizables en los principales catálogos de bibliotecas. En el caso de las revistas existen importantes directorios anglosajones de revistas por materias, aunque recogen muy pocas revistas españolas y menos de Historia. Pensemos que actualmente se publican en papel en nuestro país más de 300 revistas de Historia, contando con las llamadas misceláneas y de estudios locales, donde publican también un gran número de historiadores. La mayoría tienen además su página en Internet, y en muchos casos incorporan los sumarios de números y volúmenes.

Cabe hacer una distinción entre ediciones electrónicas de revistas que previamente son editadas en papel y revistas electrónicas "puras», concebidas para ser difundidas a través de la red. La edición elctrónica tiene la gran ventaja de su bajo coste, ya que la suscripción a algunas de las más importantes revistas internacionales alcanzan valores medios en torno a los 1.500 euros.

En conjunto hablamos de un negocio de unos diez mil millones de euros al año y al que se le calculan márgenes de beneficio cercanos al 30\%. Elsevier, el mayor emporio editorial tiene en su catálogo unas 2000 revistas, lo que le supone beneficios de hasta 600 millones de euros Las cifras son significativas porque hasta las instituciones ricas tienen problemas para abordar estos gastos. La Universidad de California, por ejemplo, paga por suscripciones de revistas 30 millones de euros (el 15, por cierto, es para Elsevier $)^{15}$.

Las revistas electrónicas tienen además la posiblidad de incorporar elementos de valor añadido: enlaces hipertexto a citas y otros recursos, metadatos, diversidad de formatos, etc. Además de las ventajas de almacenamiento, la comunicación bidireccional entre autor y lector o la actualización inmediata son valores añadidos.

La aparición en cada vez mayor número de revistas electrónicas de Historia $^{16}$, puede hacer cambiar este panorama en beneficio de la difusión y gratuidad de la información.

Solo un directorio, The History Journal Guide ${ }^{17}$, ofrece información sobre más de 6.000 revistas de Historia, y 245 revistas electrónicas gratuitas a texto completo.

Está claro que el camino hacia la publicación digital no tiene marcha atrás, y frente a cierta desconfianza inicial derivada de temas como los derechos de autor, la valoración de los especialistas o la inconsistencia de Internet, está primando el

15 Cindoc-CSIC. «Revistas científicas electrónicas: estado del arte»E-Revist@as. 2004, pag. 19 http://www.tecnociencia.es/e-revistas/especiales/revistas/pdf/e-revistas_informe.pdf (Leído el 10-62005)

16 Incluímos en el apéndice una lista de las revistas electrónicas españolas de Historia.

17 http://www.history-journals.de/ 
ahorro de sus costes de producción, lo cual es un aspecto fundamental en esta disciplina, donde muchas revistas en papel mueren por falta de presupuesto. A ello debe añadirse una mayor difusión de los trabajos y la garantía de las instituciones editoras respecto a la calidad, que cuando son universidades o centros de investigación aprovechan su infraestructura informática. Hoy conviven un buen número de empresas proveedoras que a través de sus directorios ofrecen, previo password y/o pago los artículos en línea a texto completo, con plataformas, portales y servicios que alojan las revistas electrónicas de forma abierta y gratuita.

\section{El acCeso a las Tesis doctorales y ACtas de Congresos}

Siendo la tesis doctoral el más importante recurso de información bibliográfica para los especialistas, sería muy interesante una mayor accesibilidad y disponibilidad de los textos completos. En España existen algunos intentos de universidades e instituciones, como TDC@ $T^{18}$ la red de tesis a texto completo de las universidades catalanas. El Instituto Cervantes ofrece también su sede virtual para las tesis en lengua castellana.

Además de TESEO ${ }^{19}$, que recoge las referencias de de tesis españolas, el principal repertorio internacional es DISSERTATION ABSTRACTS ${ }^{20}$ de la UMI (University Microfilms) de Ann Arbor (Michigan), que aunque nació en 1861 con voluntad de ser repositorio de tesis americanas, en la actualidad tiene una proyección internacional, aunque recoge pocas tesis españolas.

Congresos, Conferencias y Mesas Redondas, cuyas actas no se publican generalmente y solo en ocasiones aparecen, con bastante retraso, como monografías, números monográficos de revistas o parcialmente alguna ponencia como artículo de revista, tienen presencia muy dispersa en la red. En muchos casos solo se obtiene la reseña de su celebración y es casi imposible detectar a través de los catálogos y bases de datos las ponencias y sus autores.

Las Actas de Congresos publicadas en forma de libro están accesibles aunque de forma unitaria en los catálogos de Bibliotecas. También la base de datos ISOC-Historia recoge selectivamente algunas ponencias. Desgraciadamente no existe aún ninguna base de datos que cubra de forma sistemática y analítica dichas publicaciones.

\section{BASES DE DATOS BIBLIOGRÁFICAS DE HISTORIA}

Nos referimos en este caso al conjunto de referencias bibliográficas sobre una materia y cuyos registros no son accesibles a través de los simples buscado-

\footnotetext{
18 http://www.tdcat.cesca.es/

19 www.mec.es

20 http://wwwlib.umi.com/dissertations
} 
res de Internet. Permiten la recuperación por campos y en algunos casos tienen índices de contenido, materias o descriptores, identificadores, topónimos, etc.

El directorio "Internet Invisible» ${ }^{21}$ recoge más de 2.500 bases de estas características, entre ellas 39 de Historia. Si accedemos a Complete Planet, ${ }^{22}$ que ofrece más de 70.000 bases de datos, bajo el epígrafe History encontramos 192 entradas, de muy diversa tipología, pero muy pocos de ellos contienen información bibliográfica.

Dentro de las Bases de Datos bibliográficas de Historia, podemos distinguir varios tipos:

- Bases de datos multidisciplares. En ellas podemos encontrar además trabajos de otras disciplinas de las Humanidades o Ciencias Sociales. Un ejemplo es la Base de Datos ISOC, que permite el acceso conjunto a las distintas sub-bases de Humanidades, aspecto importante en una disciplina como la Historia, con relaciones temáticas a otras Ciencias Sociales.

- Bases de datos generales, con variada tipología documental: monografías, compilaciones, artículos de revistas o actas de congresos. La mayoría son de temática especializada como la alemana Regesta Imperi ${ }^{23}$ que centrada en la Edad Media recoge una importantísima cantidad de información sobre el medievalismo publicado en España.

- Bases de datos de artículos de revistas. Estas últimas pueden ser descriptivas, recogiendo solo los sumarios de las revistas, como Dialnet y por tanto permiten solo la búsqueda por título del artículo o por nombre de revista o bases de datos analíticas con análisis de contenido como IsocHistoria $^{24}$, o Historical Abstracts.

Ninguna de las dos, fundamentales para acceder a los artículos de revistas, están accesibles de forma gratuita en Internet. La primera solo es consultable en forma reducida, a partir de los sumarios, y lo mismo que Historical Abstracts, la versión completa es consultable mediante contrato. Sería de desear que también las grandes bases de datos bibliográficas, sobre todo aquellas creadas por instituciones públicas, fueran de acceso gratuito.

- Bases de datos de citas. Con una finalidad concreta, además de la informativa, las bases de datos de citas, son además de fuente de información, un recurso para conocer el impacto y la difusión de autores y publicaciones. La más conocida internacionalmente es Citation Index ${ }^{25}$, utilizada a pesar de su alto sesgo anglosajón como sistema para medir el impacto y la visibi-

21 http://www.internetinvisible.com

22 http://www.completeplanet.com

23 http://www.regesta-imperii.org/

24 http://ebano.csic.es:8085/index.jsp

25 La consulta al ISI puede realizarse a través de las bibliotecas universitarias o la biblioteca virtual del CSIC en el «Web of Knowledge» 
lidad de la producción científica española. Otro ejemplo de base de datos de citas española, más próxima a la realidad historiográfica nacional, es ModernitasCitas, de Historia Moderna, en proceso de elaboración.

En comparación con otras disciplinas, la oferta de Bases de Datos de Historia en Internet es aún escasa, de manera que en algunas áreas los recursos son insuficientes o muy actuales y dado el carácter acumulativo de esta disciplina debemos seguir recurriendo a las bibliografías retrospectivas en papel para obtener una información completa.

Muchas de las grandes bases de datos de Historia no son gratuitas, pero su consulta es posible desde las bibliotecas virtuales universitarias y de centros de investigación como el CSIC, que las hacen accesibles de forma restringida a su comunidad científica. Entre otras citamos la Biblioteca virtual del CSIC, que desde Mayo de 2005, permite desde un único punto la consulta conjunta a catálogos y bases de datos, acceso a texto completo de revistas, descarga de registros, solicitud de fotocopias y préstamo, etc.

El catálogo del distribuidor Dialog que recoge 450 Bases de Datos solo tiene 13 Bases de Humanidades, entre multidisciplinares y específicas y solo dos de Historia: Historical Abstracts y America History $\mathcal{E}$ Life. En ellas hay una escasa presencia de publicaciones españolas. Historical Abstracts recoge 2.427 revistas publicadas en 90 paises, de las que solo 27 son españolas. A pesar de todo en los últimos años ha habido un importante crecimiento en la aparición de bases de datos especializadas por épocas. El carácter interdisciplinar de la Historia, obliga además a consultar un buen número de bases de datos y bibliografías de otras disciplinas cercanas.

En cualquier caso no hay que olvidar la proliferación de pequeñas bases de datos existentes en Departamentos de Universidades y sedes de Comunidades Autónomas, sobre Historia por épocas, regiones ó temas, aunque muchas de ellas están faltas de cobertura y actualización.

El mayor avance en las bases de datos de artículos de revistas, es el gran número de enlaces que establecen no solo a los webs de las revistas sino a los textos completos.

\section{BIBLIOGRAFIAS ESPECIALIZADAS EN INTERNET}

La cantidad de bibliografías especializadas, no solo por épocas, sino por temas o acontecimientos en Internet es innumerable. Ubicadas en webs institucionales, páginas personales de profesores, o en programas de departamentos, resultan útiles siempre que tengan una cierta exhaustividad o calidad en la selección, a pesar de su indudable dispersión. En el apéndice final recogemos algunos ejemplos como muestra. Un aspecto muy interesante es lo que ofrece el propio buscador de Google, permitiendo acceder a texto completo a un buen 
número de monografías de historia publicadas en inglés. Suelen permitir visualizar de forma gratuita todas las páginas de la bibliografía adjunta.

\section{CONCLUSIONES}

Después de lo expuesto, podemos afirmar que el futuro del acceso a la información bibliográfica ha dado en los últimos años un salto cuantitativo y cualitativo gracias a Internet. Las dificultades contrastadas hace diez años en la elaboración de bibliografías especializadas, se han reducido enormemente. Aún quedan por solucionar problemas como el acceso y localización de muchos documentos reseñados, debido a que algunas bibliotecas y centros de información que ofrecen sus catálogos en Internet, no prestan ni facilitan originales o fotocopias.

Otras, sin embargo, han creado Bibliotecas Virtuales, que gracias a las nuevas tecnologías, permiten un acceso global a los recursos electrónicos e impresos, tanto de suscripción como gratuitos. El usuario puede lanzar búsquedas temáticas a varios recursos a la vez, y disponer de servicios de acceso al texto completo o solicitud en línea de los documentos.

El carácter acumulativo y falto de obsolescencia de la historiografía hace aún necesario acudir a repertorios bibliográficos en papel para la búsqueda de información retrospectiva. Hay grandes catálogos y bibliografías de obligada consulta que aún no están en Internet, como el Palau, Indice Histórico Español, Bibliografía General Española, International Medieval Bibliography, y así hasta un larguísimo etcétera.

Desde luego el avance más importante que ofrece Internet está en la digitalización y edición electrónica de documentos con la creación de grandes repositorios a texto completo y la presencia cada vez mayor de los servicios gratuitos de acceso a revistas electrónicas o cualquier otro tipo de publicación. Por encima de prejuicios inconsistentes relativos a la defensa de los derechos de autor, hoy superados por las garantías legislativas, la persistente falta de valoración institucional y docente de las publicaciones eléctrónicas, hace que muchos investigadores, preocupados por su currículo profesional, sean reacios a publicar en este medio. Pero las ventajas y posibilidades de la edición electrónica son incuestionables, y el tiempo hará superar estos impedimentos.

La iniciativa Open Access intenta que en un futuro próximo los usuarios de Internet puedan leer, descargar, copiar, distribuir, imprimir, buscar, o enlazar los textos completos de los artículos científicos, y, usarlos con cualquier otro propósito legítimo, sin otras barreras financieras, legales o técnicas que las que suponga Internet en sí misma. Es decir, sin coste alguno, y otorgando a los propios autores el control sobre la integridad de su trabajo y el derecho a ser adecuadamente reconocidos y citados.

En cualquier caso, el futuro de Internet depende por supuesto de la implicación y participación de la comunidad de historiadores. Los productos biblio- 
gráficos elaborados por los propios especialistas y documentalistas con formación histórica tendrán siempre una mayor garantía de calidad, por poseer éstos un mayor conocimiento de las necesidades reales de información, y una mayor capacidad de valoración de los documentos.

\section{DIRECCIONES DE RECURSOS BIBLIOGRÁFICOS DE HISTORIA EN INTERNET}

\section{El acCeso a los libros. Catálogos de bibliotecas}

\subsection{Directorios de Bibliotecas}

\section{Internacionales}

The Library Index

http://www.libdex.com

Ofrece enlace a 18.000 bibliotecas

Library Planet

http://libraryplanet.com

\section{España}

http://rediris.es/recursos/bibliotecas

Directorio de la Red Iris

http://exlibris.usal.es/bibesp

Buen directorio de Biblioteca de la Universidad de Salamanca

http://cervantesvirtual.com/

Bibliotecas del Mundo del Instituto Cervantes

http://www.csic.es/cbic

Directorio del CSIC

1.2. Catálogos y bibliografías nacionales.

\section{España}

ARIADNA. Catálogo de la Biblioteca Nacional de España http://www.bne.es

Catálogo Colectivo del Patrimonio Bibliográfico Español www.mcu.es/ccpb

Obras anteriores al siglo XIX

Catálogo de la Agencia Española del ISBN

www.mcu.es/bases/spa/isbn/ISBN.html 
Otros países

OPALE. Catálogo de la Biblioteca Nacional de Francia http://www.bnf.fr

Catálogo de la Biblioteca Alemana www.ddb.de

Catálogo de la Biblioteca Nacional Central de Florencia www.bncf.firenze.sbn.it

Catálogo de la Biblioteca Nacional de Portugal www.porbase.bn.pt

Catálogo de la Biblioteca del Congreso de Washington www.loc.gov

\section{British Library}

http://www.bl.uk/

\subsection{Catálogos colectivos}

\section{España}

Catálogo Colectivo de las Bibliotecas del CSIC www.csic.es/cbic/cbic.htm

\section{REBIUN}

http://www.uma.es/rebiun/

Participan más de 50 bibliotecas universitarias

\section{CBUC}

http://www.cbuc.es/

Catálogo Colectivo de las Universidades de Cataluña

\section{Fons d'Història Local}

http://www.diba.es/fonshl/fonshl.asp?cont $=157$

Diputación de Barcelona - Universidad Autónoma de Barcelona, Dpto. de Historia Moderna y Contemporánea. Catálogo de libros fundamental para la Historia de Cataluña

\section{Catálogo colectivo de Euskadi}

http://www.liburutegiak.euskadi.net/cgi-bin_q81a/bilgunea/X9486/

ID965781624?ACC $=101$ 
Otros países

\section{SUDOC}

www.sudoc.abes.fr

Catálogo del Systeme Universitaire de Documentation (SUDOC) de l'Agence Bibliographique de l'Enseignement Superieur (Francia). Acoge los catálogos de la mayoría de las bibliotecas universitarias francesas

\section{COPAC}

http://www.copac.ac.uk/

Copac. Catálogo colectivo de bibliotecas universitarias inglesas

\section{CIC}

http://cicvel.lib.uiowa.edu/

Catalogo colectivo de Universidades norteamericanas. Solo accesible mediante password

\section{KVK}

http://www.ubka.uni-karlsruhe.de/kvk/kvk/kvk_fr.html

Metacatálogo de la Universidad de Karlsuhe. Recoge 76 millones de libros. Además de permitir la búsqueda simultánea en los catálogos colectivos regionales alemanes, permite interrogar a las principales bibliotecas nacionales del mundo

\section{SBN}

http://opac.sbn.it/cgi-bin/IccuForm.pl?form = WebFrame

Opac del Servicio Bibliotecario Nacional italiano, que utiliza el protocolo Z39-50 para el acceso conjunto a las bibliotecas de Italia

\subsection{Otros catálogos españoles con importantes fondos de Historia}

\section{Universidad Complutense (CISNE)}

http://www.ucm.es/BUCM

\section{Universidad Autónoma de Madrid}

www.biblioteca.uam.es

\section{UNED}

http://www.uned.es

\section{Universidad de Barcelona}

http://www.ub.es

\section{Universidad Autónoma de Barcelona}

http://www.bib.uab.es/internet.htm 


\section{Universidad de Salamanca}

http://www.usal.es

Universidad de Valencia

http://uv.es/biblios

Universidad de Santiago de Compostela

http://www.usc.es

Universidad de Sevilla

http://www.us.es

Universidad País Vasco

http://hermes.lp.ehu.es

Arteleku.

http://www.arteleku.net/secciones/documental/biblioteca/biblioteca.html Biblioteca on-line de la Diputación de Guipúzcoa. Interesante para la Historia del País Vasco

\section{Fundación Sancho el Sabio}

http://www.fsancho-sabio.es/inicio.htm

Además de su catálogo, presenta una biblioteca digital de autores vascos

\section{Centro de Estudios de Castilla-La Mancha}

http://www.uclm.es/Ceclm/indexr.htm

Ofrece además de su catálogo, una bibliografía regional con vaciado de revistas, compilaciones, tesis, etc. Otra base de datos de documentos a texto completo y dos bibliografías especializadas sobre Toledo y las Brigadas Internacionales

\section{CatÁlogos Comerciales}

http://bookwire.com

Guía de recursos del mundo del libro: editores, libreros y bibliotecas

http://www.marcialpons.es

De especial interés por sus importantes colecciones de Historia. Ofrece además bibliografías temáticas.

http://www.leer.nisc.com/

Catálogo que permite localizar libros no solo disponibles sino agotados

\section{Iberlibro}

http://www.iberlibro.com/ 
Catálogo colectivo de libros en venta de España y Portugal. La mejor librería virtual de antiguo y ocasión en castellano. Más de 2.000.000 de libros

\section{Libros con Historia}

http://www.librosconhistoria.com/

Librería de viejo especializada en Historia

http://onlinebooks.library.upenn.edu/authors.html

Cientos de libros clásicos y actuales sobre Historia

\section{Amazon}

http://www.

Hipermercado del libro. Permite hojear los indices y primeros capítulos

\section{Libros Reyes}

http://www.librosreyes.com/7cec834fc46dfa1031735c5a4ac1f952/

Librería de Historia

\section{E-BOOKS Y REPOSITORIOS DE DOCUMENTOS ELECTRONICOS}

\section{E-libro}

http://www.e-libro.com/

El mejor sitio de libros electrónicos en español.

\section{Reti Medievali}

http://www.storia.unifi.it/ RM/RM-Home-es.htm

Creado por la Univ. de Florencia recoge ensayos, textos y trabajos de historiografía medieval.

\section{AHDS History}

http://hds.essex.ac.uk/

Repositorio de recursos digitales para la investigación y la enseñanza de la Historia

\section{Humbul}

http://www.humbul.ac.uk/output/shortout.php?no = 180\&type $1=$ seco ndary\&type $2=\mathrm{XXXX} \&$ subj $=$ history\&ref $=$ subout Este sitio recoge gran número de recursos históricos, tanto primarios como secundarios.

\section{REPEC}

http://repec.org/

Repositorio de documentos de interés para la Historia económica. Documentos de trabajo, artículos, libros etc. 


\section{The Library of Iberian Resources Online (LIBRO)}

http://libro.uca.edu/

Proyecto sobre Historia medieval de España de la Univ. de Arkansas, presenta a texto completo un importante número de monografías publicadas por hispanistas de universidades americanas

\section{Questia}

http://www.questia.com/library/history/

Más de 50.000 libros y 400.000 artículos de revistas a texto completo de Humanidades y Ciencias Sociales. El apartado de Historia, tiene una organización cronológica y temática muy interesante, aunque con claro predominio de publicaciones anglosajones.

\section{El ACCESO A LAS REVISTAS}

\subsection{Directorios de revistas de Historia}

\section{Electronic Journals Resource Directory}

http://library.usask.ca/ scottp/links/

Enlaza con directorios, bibliotecas y servidores de revistas electronicas

\section{Catalog of Electronic Journal}

http://www.edoc.com/ejournal

Lista internacional de revistas electrónicas por materias.

http://ns.fcs.ucr.ac.cr/ historia/enlaces/e-revis.htm

Lista de revistas electrónicas de Historia

\section{The History Journals Guide}

http://www.history-journals.de/

Creado por la Virtual Library History de La Universidad de Florencia. Contiene un directorio de 6.300 revistas de Historia, incluyendo 220 revistas electrónicas a texto completo y 20.000 reseñas bibliográficas de otras fuentes primarias y secundarias.

\section{Project Muse}

http://muse.jhu.edu/journals/subject.html\# history

Más de 100 revistas de Historia, del ámbito anglosajón a texto completo. Permite búsqueda por titulo del artículo, materia y texto libre.

\section{Doaj}

http://www.doaj.org/ljbs?cpid $=11$

Directorio Open Access de artículos. Organizado por materias, contiene 40 revistas electrónicas de Historia. 


\section{JSTOR}

http://www.jstror.org

Directorio de revistas con más de 100 revistas anglosajonas a texto completo

\subsection{Revistas electrónicas españolas de Historia}

\section{Hispania Nova. Revista de Historia Contemporánea}

http://hispanianova.rediris.es

Revista de Historia fundada por el Prof. Angel Martínez de Velasco. Dirigida por Julio Aróstegui (Univ. Complutense) y Esteban Canales (Univ,. Autónoma de Barcelona)

\section{Clío}

http://clio.rediris.es

Revista de Historia, dirigida a poner en la red materiales didácticos para la enseñanza de la Historia y potenciar el uso de Internet entre los historiadores.

\section{Revista de Estudios Ibéricos}

http://www.ffil.uam.es/reib/

Revista de difusión de la Cultura Ibérica

\section{Revista de Historia Constitucional}

http://constitucion.rediris.es/revista/hc/index.html

Creada por el Dpto. de Derecho de la Universidad de Oviedo

\section{Tiempos Modernos}

http://tiemposmodernos.rediris.es/

Revista de Historia Moderna. Utiliza el sistema Open Access.

\section{TESIS DOCTORALES Y ACTAS DE CONGRESOS}

\section{SIGLE}

Sistema de Información de Literatura Gris. Base de datos multidisciplinar que contiene tesis doctorales, actas de congresos $\mathrm{Y}$ documentos de trabajo. Distribuido por WSpirs tiene acceso gratuito para los usuarios de muchas bibliotecas.

\section{Proquest digital dissertations}

http://wwwlib.umi.com/dissertations

El Dissertations Abstracts ofrece en línea de forma gratuita las tesis recogidas por la UMI, desde 1997. 


\section{REDIAL-TESIS}

http://pci204.cindoc.csic.es/Htdocs/cindoc/tesis.htm

Tesis europeas sobre América Latina

\section{TESEO}

www.mec.es

El Ministerio de Educación español en su base Teseo recoge referencias de las tesis españolas a partir de 1975, aunque existe bastante retraso en su introducción.

\section{CISNE-TESIS}

www.cisne.sim.ucm.es

Este catálogo contiene más de 7.000 tesis a texto completo, leídas en la UCM entre 1990 y 2004, entre ellas unas 500 de Historia

\section{TDC@T}

http://www.tdcat.cesca.es/

Tesis doctorales a texto completo leídas en universidades catalanas.

\section{FAMA}

http://fama.us.es/search*spi S3

Catálogo de tesis de la Universidad de Sevilla

\section{Biblioteca Virtual Cervantes. Tesis doctorales}

http://www.cervantesvirtual.com/tesis/tesis_catalogo.shtml

Edición digital de tesis leídas en 44 universidades.

\section{BASES DE DATOS BIBLIOGRAFICAS}

\subsection{Directorios de Bases de Datos}

http://www.library.upenn.edu/resources/databases

Webb de la Univ. de Pennsilvanya, para acceso a bases de datos

\section{Internet Invisible}

Para conocer cuales son las principales bases de datos gratuitas en Internet http://www.internetinvisible.com

\subsection{Bases de datos de sumarios de revistas}

\section{DIALNET}

http://dialnet.unirioja.es/

Base de datos de sumarios elaborada por la Universidad de la Rioja, contiene un número importante de revistas de Historia, además de artículos de compilaciones. Permite la búsqueda por título de la revista y del artículo, algunos a texto completo. 


\section{Periodicals Index Online}

http://pio.chadwyck.co.uk/home.do

Acceso restringido. Recoge 12 millones de artículos de revistas en Ciencias Sociales y Humanidades aparecidas desde 1770, algunas de ellas a texto completo. Dada la antigüedad de las revistas recogidas representa un importante fondo de archivo para los historiadores.

\section{Consorcio de Bibliotecas Universitarias de Cataluña}

http://sumaris.cbuc.es/

Acceso a los sumarios electrónicos de revistas, con 200.000 sumarios correspondientes a 10.000 títulos de revistas. De ellas más de 200 revistas son de Historia.

\section{Article@inist}

http://services.inist.fr/public/fre/conslt.htm

Base de datos de monografías, artículos de revistas y actas de Congresos, elaborada por el Institut d'Information Cientifique et Tecnique del CNRS (Francia)

\subsection{Bases de datos analiticas de articulos de revistas}

\section{Isoc-Historia (CINDOC - CSIC).}

http://www.cindoc.csic.es

Elaborada por el Cindoc (CSIC) es la base de datos de artículos de revistas más importante para la Historia de España. Recoge bibliografía desde 1975 hasta hoy, y actualmente contiene más de 50.000 referencias. Se presenta en dos formatos: Base de datos analítica accesible mediante password y gratuita de sumarios.

\section{Historical Abstracts}

Recoge artículos de 2.427 revistas publicadas en 90 países y en 30 lenguas.Tiene dos series:
A) Modern History Abstracts. 1450 a 1914
B) Twenttieth-Century Abstracts. De 1914 a hoy

\section{America - History \& Life}

Creada por el mismo editor de la anterior, recoge información sobre la Historia de Estados Unidos

\section{Hapi.-Hispanic American Periodicals Index}

http://hapi.gseis.ucla.edu/

Revistas sobre Historia de América. Acceso restringido.

\section{Handbook of Latin American Studies}

http://lcweb2.loc.gov/hlas/

Editada por la División Hispánica de la Biblioteca del Congreso. Acceso gratuito. 


\subsection{Bases de datos de citas}

ISI. Web of KnowledgeA

http://isiknowledge.com/

Incluye Arts RTS\&HUMANITIES CITATION INDEX y SOCIAL CITATION INDEX

Permite conocer los autores citados en las revistas. Predominio de revistas anglosajonas.

\section{Modernitas Citas}

http://www.dge.filol.csic.es/emc/

Base de datos de citas obtenidas de artículos de revistas y actas de Congreso sobre Historia de España Moderna.

\section{OTRAS BASES DE DATOS ESPECIALIZADAS}

\section{Regesta Imperii}

http://regesta-imperii.uni-giessen.de/lang_en/index.php

Bases de datos alemana que recoge documentos primarios y bibliografía sobre la Historia Medieal de Europa

\section{Index Islamicus}

http://www.csa.com/factsheets/islamicus-set-c.php

Base de datos de artículos de revista sobre el mundo islámico desde la Edad Media hasta la actualidad.

\section{BAHR. Bulletin Analitique d' Histoire romaine}

http://argentoratum.u-strasbg.fr/portail-poucet/12.bahr.htm

Base de datos del mundo romano elaborada por la Universidad de Estrasburgo

\section{AnPhilNet.L'Anne Philologique en línea}

http://www.aph.cnrs.fr/

Base de datos bibliográfica sobre el mundo greco-latino

\section{Repertorio de Medievalismo Hispanico}

http://www.imf.csic.es/medieval/busqueda.htm

Información bibliográfica sobre historia medieval y musicología, elaborada por el Instituto Milá y Fontanals del CSIC.

Modernitas: Base de datos bibliográfica sobre historia moderna.

http://clio.rediris.es/clionet/biblos/modernitas.htm

Base de datos de Historia Moderna elaborada por Francisco Fernandez Izquierdo del CSIC.

\section{Internacional Women's Resources.}

http://www.nisc.com.mx/productos/wri_html Bases de datos sobre Historia de Género de la Universidad de Madison (USA) 


\section{TOCS-IN}

http://www.chass.utoronto.ca/amphoras/tocs.html

Base de datos de artículos de revistas y actas de Congresos sobre estudios clasicos.contiene 185 revistas y 45.000 artículos. Credad por la Univ. de Toronto.

\section{ALGUNAS BIBLIOGRAFÍAS EN LA RED}

http://print.google.com

El buscador de Google permite el acceso a la bibliografía de un gran número de libros de Historia

http://seneca.uab.es/historia/do5bib.htm

En la página de Esteban Canales, encontramos páginas de cursos de Contemporánea con una interesante bibliografía sobre la Guerra de la Independencia

\section{Bibliografía de Historia de Portugal en la Red} http://www.uc.pt/BAHP/bahp90.arq.top.html

Bibliografía sobre el 98

http://home.coqui.net/sarrasin/bibliografia.htm\# anchor 415690

\section{Bibliografías de Historia Antigua}

http://155.210.60.15/HAnt/Bibliografias/index.html

Páginas del Dep. de Historia Antigua de la Universidad de Zaragoza con recopilaciones bibliográficas sobre distintos aspectos de la Historia de España antigua http://www.ucm.es/info/hisdere/pbporras.htm

Bibliografías sobre Historia del Derecho y Fuentes medievales, elaborada por Pedro Andrés Porras

\section{Bibliografía de Historia de Internet}

http://www.aui.es/historia/

Como demostración de que Internet ya es Historia, esta página nos muestra una recopilación de referencias bibliográficas sobre el tema.

\section{BIBLIOGRAFÍA}

CANALES, Esteban. «La Historia en la red». Fer história des dels mitjans de comunicació $i$ les noves tecnologies, Curso organizado por la UAB, el día 13 de junio del 2003.

En: http://seneca.uab.es/historia/red/index.htm (Leído el 10-3-2005).

BARATAS DíAZ, Luis Alfredo Y FERNÁNDEZ PÉREZ, Joaquín: «Internet: un recurso imprescindible para historiadores de la ciencia y la tecnología», Llull, 1995, vol. 35, n 35, pp. 667-675. 